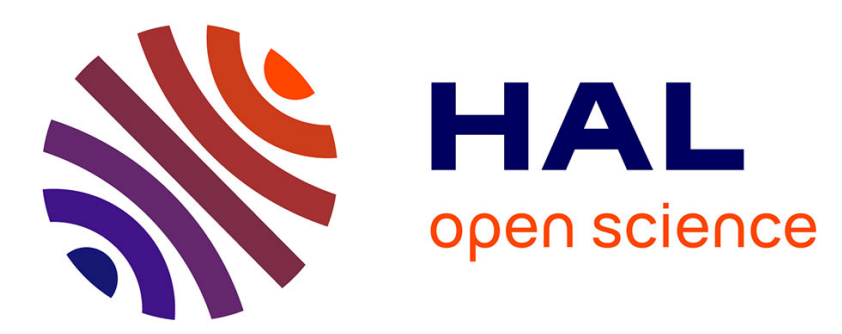

\title{
Temperature dependence of quantum dot fluorescence assisted by plasmonic nanoantennas
}

\author{
Q. Le-Van, X. Le Roux, T. V. V Teperik, B. Habert, F. Marquier, J.-J.
}

Greffet, A. Degiron

\section{- To cite this version:}

Q. Le-Van, X. Le Roux, T. V. V Teperik, B. Habert, F. Marquier, et al.. Temperature dependence of quantum dot fluorescence assisted by plasmonic nanoantennas. Physical Review B: Condensed Matter and Materials Physics (1998-2015), 2015, 91 (8), pp.85412 10.1103/PhysRevB.91.085412 . hal-01636515

\section{HAL Id: hal-01636515 \\ https://hal.science/hal-01636515}

Submitted on 17 Nov 2017

HAL is a multi-disciplinary open access archive for the deposit and dissemination of scientific research documents, whether they are published or not. The documents may come from teaching and research institutions in France or abroad, or from public or private research centers.
L'archive ouverte pluridisciplinaire HAL, est destinée au dépôt et à la diffusion de documents scientifiques de niveau recherche, publiés ou non, émanant des établissements d'enseignement et de recherche français ou étrangers, des laboratoires publics ou privés. 


\title{
Temperature dependence of quantum dot fluorescence assisted by plasmonic nanoantennas
}

\author{
Q. Le-Van, ${ }^{1}$ X. Le Roux, ${ }^{1}$ T. V. Teperik, ${ }^{1}$ B. Habert, ${ }^{2}$ F. Marquier, ${ }^{2}$ J.-J. Greffet, ${ }^{2}$ and A. Degiron ${ }^{1, *}$ \\ ${ }^{1}$ Institut d'Electronique Fondamentale, Université Paris-Sud and CNRS, UMR 8622, Orsay F-91405, France \\ ${ }^{2}$ Laboratoire Charles Fabry, Institut d'Optique, CNRS, Université Paris-Sud, 2 avenue Fresnel, 91127 Palaiseau cedex, France
}

(Received 5 September 2014; revised manuscript received 27 January 2015; published 12 February 2015)

\begin{abstract}
Optical antennas based on noble metal nanoparticles can increase the photoluminescence of quantum dots, but the exact strength of this enhancement depends on the brightness (i.e., the intrinsic quantum yield $\eta_{i}$ ) of the emitters. Here we perform temperature-dependent measurements on a system of $\mathrm{PbS}$ colloidal quantum dots coupled with Au ring arrays that bring quantitative insight into this phenomenon. We show that although the boost in photoluminescence is lower at cryogenic temperatures where the nanocrystals become very bright emitters, the spectral signature of this enhancement is remarkably independent of $\eta_{i}$. These observations remain true even at wavelengths where the losses by absorption in the metal nanoparticles considerably increase due to the excitation of localized plasmon resonances, in contradiction with standard theory that treats the emitters as a collection of two-level systems. We propose a mechanism in which the quantum dots are modeled as multilevel and inhomogeneously broadened emitters to account for these findings.
\end{abstract}

DOI: 10.1103/PhysRevB.91.085412

PACS number(s): 73.20.Mf, 78.67.-n, 81.07.Ta, 82.35.Np

A metal nanoparticle can be used as an optical antenna that controls how light is absorbed by and/or extracted from a quantum emitter [1]. The most efficient antennas are those that become resonant through the excitation of localized surface plasmons, resulting in dramatic modifications of the intensity and spatial distribution of the emitted light [2-13]. Some of the best experimental implementations reported so far are subwavelength plasmonic dimers that increase the rate of spontaneous emission by several orders of magnitude [6-8], while larger antennas can transform the dipolar distribution typical of point-source emitters into highly directive patterns [11-13]. In parallel to these advances on individual antennas, the related problem of emitters coupled with random and periodic arrangements of plasmonic nanoparticles has also been investigated for a long time [14-20].

The majority of these photoluminescence (PL) experiments can be interpreted within a semianalytical framework whereby the emitter is treated as a two-level system characterized by a classical dipole moment. This approach makes it possible to account for the salient features of the plasmon-assisted PL, i.e., a modification of the pumping conditions at the excitation frequency, an increase in the rate of spontaneous emission at the fluorescence wavelength, as well as changes in the far-field characteristics of the emitted light. However, it has also been pointed out that a number of experimental configurations cannot be fully understood with this generic model $[9,21,22]$. Such is the case for instance with experiments involving fluorescent molecules that can radiatively relax to a vibrational ground state manifold: The transitions that are energetically matched with the plasmonic resonance frequency are selectively enhanced, producing a PL enhancement distinct from the predictions of the two-level system [21]. Interestingly, similar spectral signatures have been observed in PL experiments involving inhomogeneous distributions of semiconducting nanocrystals [20], suggesting that plasmonic antennas also favor preferential radiative transitions

\footnotetext{
*aloyse.degiron@u-psud.fr
}

in colloidal quantum dots (CQDs) and quantum rods. This possibility deserves to be thoroughly investigated because the fluorescence of molecules and the PL of quantum dots obey to very different mechanisms: while the former arises from the relaxation to a molecular orbital of lower energy, the latter corresponds to the recombination of an exchange-correlated exciton whose energy structure and spin properties have no direct equivalent in molecules. The optical properties of CQDs are further complicated by anisotropies in their shape, the existence of trap states and emitting defect states at the surface of the nanocrystals, the possibility of multiexciton formation, and the fact that not all excitons have an angular momentum that allow them to directly couple with light [23]. In addition, an ensemble of CQDs is always subject to a large inhomogeneous broadening, which is in contrast with molecules where the importance of this parameter depends on the chemical environment and the time scales involved in the experiments [24].

In this article we examine and clarify the coupling between plasmonic antennas and colloidal quantum dots embedded in a dielectric matrix. With a series of temperature-dependent experiments performed under weak continuous wave excitation (i.e., below saturation level), we show that the spectral shape of the PL enhancement does not depend on the intrinsic quantum yield $\eta_{i}$ of the emitter. This result implies that the efficiency of lossy plasmonic antennas remains high even when they are coupled with an ensemble of bright CQDs characterized by near-unity $\eta_{i}$. We show that our measurements cannot be understood if one depicts the CQDs as two-level emitters and propose a theoretical model that treats the CQDs as an inhomogeneous collection of multilevel sources to support our experimental findings.

One possible route to tune $\eta_{i}$ consists in working with varying concentrations of the same emitter species: as the concentration increases, reabsorption and nonradiative energy transfers between the sources become dominant, lowering their intrinsic quantum yield. This approach has been used, for example, to study the fluorescence of molecules above island metal films [15]. It is also possible to act upon $\eta_{i}$ without 
(a)

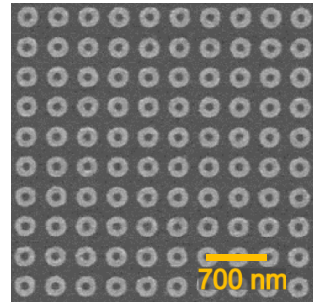

(b)

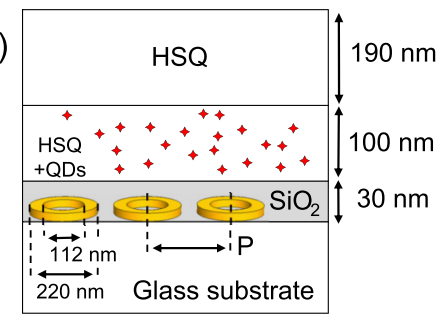

FIG. 1. (Color online) (a) SEM viewgraph of a representative ring array studied in this work (period $P=350 \mathrm{~nm}$ ). The scale bar indicates $700 \mathrm{~nm}$. (b) Schematic of the structures.

modifying the incident conditions, for example by varying the temperature of the system [25-27]. This is the strategy followed in the present study.

Figure 1 provides a description of the structures under consideration. A series of Au ring arrays with subwavelength periods ranging from 350 to $700 \mathrm{~nm}$ is patterned on a glass substrate by e-beam lithography and metal deposition. The dimensions of the rings are the same for all structures; they are characterized by an inner radius of $56 \pm 3 \mathrm{~nm}$, an outer radius of $110 \pm 2 \mathrm{~nm}$, and a metal thickness of $35 \pm 2 \mathrm{~nm}$ that includes a thin adhesion layer of Ti between Au and the substrate. The sample is subsequently coated with a stack of three layers: a spacer $(30 \mathrm{~nm})$ of $\mathrm{SiO}_{2}$ that prevents direct

\section{Energy (eV)}

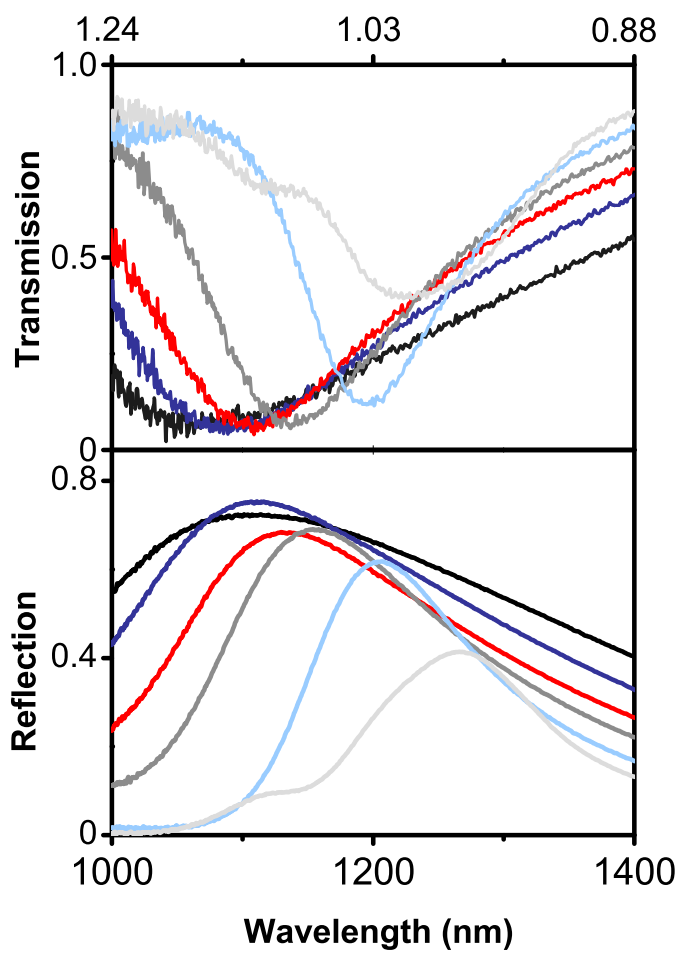

FIG. 2. (Color online) Far-field reflection and transmission spectra obtained with a Varian FTIR spectrometer coupled to an optical microscope at room temperature. Light gray: $P=700 \mathrm{~nm}$, light blue (slightly darker gray): $P=600 \mathrm{~nm}$, medium gray: $P=500 \mathrm{~nm}$, red (dark gray): $P=450 \mathrm{~nm}$, dark blue (even darker gray): $P=400 \mathrm{~nm}$, and black: $P=350 \mathrm{~nm}$. adsorption of the emitters on the rings, a $100 \mathrm{~nm}$ thick matrix of hydrogen silsesquioxane (HSQ) containing a concentration of $1015 \mathrm{PbS}$ CQDs per $\mathrm{cm}^{3}$, and a $190 \mathrm{~nm}$ thick layer of pure HSQ. The PbS QDs are commercial nanocrystals purchased from Evident Technologies with an averaged diameter of $5 \mathrm{~nm}$ (or $9 \mathrm{~nm}$ by taking the ligands into account). All the dielectric materials used here are silicon oxides having approximately the same refractive index of 1.5, ensuring that the Au ring arrays and the CQDs are in an almost symmetric environment.

The geometrical parameters have been chosen so that the rings sustain localized plasmonic resonances in the near infrared, i.e., at wavelengths much larger than their physical size $[28,29]$. To characterize these resonances, we have measured the far-field transmission $(T)$ and reflection $(R)$ spectra of the structures with a FTIR spectrometer (Fig. 2). In these experiments, the ring arrays are coated with the three layers depicted in Fig. 1(b), allowing direct comparison with the PL measurements described later in the text. The curves present a well-defined peak that shifts to the blue and broadens as the period decreases, indicating that the localized plasmons become more and more coupled as the rings are getting closer [30]. Note that the complex spectral shape obtained for $P=700 \mathrm{~nm}$ is an artifact from our experimental setup: The structures are illuminated with a converging beam that induces unwanted diffraction effects for this large period.

We characterize the infrared emission from our structures with the microphotoluminescence (PL) setup depicted in Fig. 3(a). The samples are placed inside a cryostat (Oxford Instrument MicrostatHires2) and pumped at $633 \mathrm{~nm}$ with a continuous wave HeNe laser through a $40 \times$ objective (Nachet, N.A. $=0.6$ ). The same objective is also used to collect the
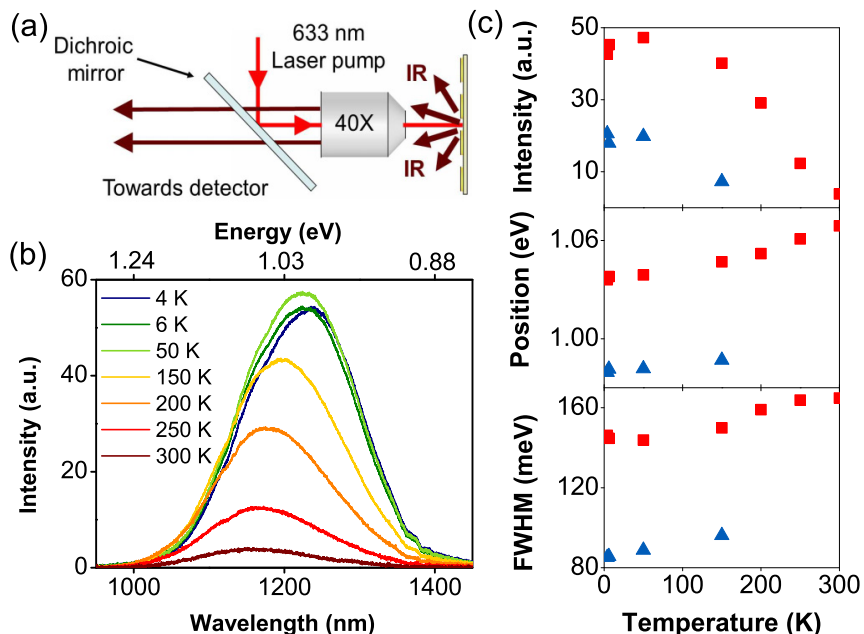

FIG. 3. (Color online) (a) Schematic of the experimental microphotoluminescence (micro PL) setup used in these experiments. Not represented in this sketch is the cryostat, a MicrostatHires 2 cold finger from Oxford Instruments. (b) Evolution of the PL spectra of the $\mathrm{PbS}$ QDs in the dielectric host matrix as a function of the temperature. The data have been taken in a region of the sample far from any metallic structure. (c) Summary of the Gaussian fit applied to the data of (b) when plotted as a function of the energy. The three panels plot the intensity, the peak energy, and the full width at half maximum (FWHM) of the Gaussian function(s) used to fit each measurement [34]. 
IR photoluminescence which is subsequently separated from the pump laser with a dichroic mirror and directed towards a monochromator (Jobin Yvon iHR320) and an InGaAs detector (Jobin-Yvon Symphony II). All the measurements of this study are performed at sufficiently low pump powers to avoid saturation of the PbS QDs and to ensure a linear relationship between pump and PL intensities at all temperatures. The distance between the sample and the microscope objective is systematically adjusted so as to maximize the PL signal that reaches the detector.

We first examine how the properties of the PbS QDs embedded in their HSQ matrix vary with the temperature $T$. For this purpose, we select a region of the sample far from any ring array and measure its luminescence for different values of $T$. The results are summarized in Fig. 3(b) which shows that the spectra feature a broad peak spanning the $1000-1400 \mathrm{~nm}$ range. As the temperature drops from 300 to $4.2 \mathrm{~K}$, this maximum is shifted to larger wavelengths, its full width at half maximum (FWHM) narrows, and its amplitude increases by a factor of 10 . This intensity enhancement can be attributed to the reduction of phonon coupling although it can be noted that the fluorescence eventually tapers off for temperatures lower than $50 \mathrm{~K}$. Such a behavior has been reported before and attributed to the splitting of the lowest energy exciton into bright and dark states, the persistence of exciton-acoustic phonon coupling at cryogenic temperatures, and the existence of trap defect states with energy barriers that become too high to be overcome at low $T$ [31,32]. Nevertheless, the quantum yield $\eta_{i}$ of CQDs in dielectric matrices approaches near unity below $50 \mathrm{~K}$ [33] and our experiments indicate that it is approximately ten times smaller at room temperature.

When plotted as a function of the energy, the same PL curves evolve from a symmetric Gaussian distribution at room temperature (typical of inhomogeneous broadening) to an asymmetric shape that is well fitted by two Gaussian functions, revealing the presence of distinct emission bands [34]. These observations, which are summarized in Fig. 3(c), are fully consistent with existing experimental data [31,35-37]. In particular, the existence of multiple emission peaks is typical of QDs embedded in a dielectric matrix because the surrounding host creates defect states that open relaxation channels at energies distinct to that of the intrinsic excitonic recombination band of the emitters [35]. Specifically, we identify the low energy peak as a defect state because its position and linewidth do not significantly vary with the temperature. In contrast, the high energy peak has the expected behavior of the intrinsic excitonic band of $\mathrm{PbS}$ nanocrystals, i.e., a redshift and narrowing that can be explained by taking into account the temperature dependence of the QD band gap, the coupling between excitons and acoustic phonons, and the interactions between excitons and LO phonons [31,35].

We now investigate how the ring arrays modify the emission properties of the CQDs. To this end, we measure the PL spectrum above each structure and normalize the data with the luminescence of a neighboring region without metallic pattern. The resulting ratio provides the PL enhancement above the metallic structures as a function of the wavelength. As before, all the measurements presented here are taken in the low pumping regime characterized by a linear dependence between the pump and PL intensities.
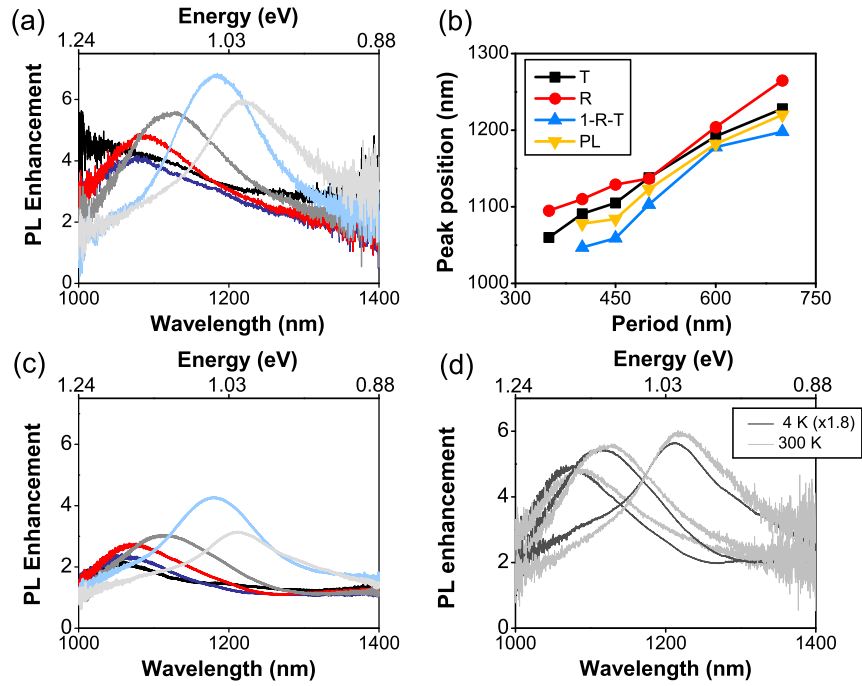

FIG. 4. (Color online) (a) PL enhancement spectra of the structures at $300 \mathrm{~K}$ for different periods of the antenna array. A PL enhancement spectrum is defined as the ratio between the PL signal recorded above a given structure and the PL signal recorded above glass away from the structure. The color scheme is the same as in Fig. 2, with curves ranging from light gray $(P=700 \mathrm{~nm})$ to black $(P=350 \mathrm{~nm})$. (b) Peak wavelengths of the transmission $T$, reflection $R$, absorption (defined as $1-R-T$ ), and PL enhancement spectra as a function of the period at $300 \mathrm{~K}$. (c) PL enhancement spectra of the structures at $4.2 \mathrm{~K}$. The color scheme is the same as in (a) and Fig. 2. (d) Comparison of the PL enhancement spectra obtained at $300 \mathrm{~K}$ with those measured at $4.2 \mathrm{~K}$ but scaled by a factor of 1.8 . Only three periods are represented for clarity: $P=450 \mathrm{~nm}, P=500 \mathrm{~nm}$, and $P=700 \mathrm{~nm}$.

Figure 4(a) summarizes the results of the measurements at room temperature. The PL enhancement spectra present the same general characteristics as the FTIR measurements, as also seen in Fig. 4(b) where we have reported the position of the different peaks as a function of the period of the array. For all the structures, the wavelength of maximum PL enhancement is comprised between the transmission and reflection peaks on one hand and the absorption peak (defined as $1-R-T$ ) on the other hand, indicating that the PbS QDs placed above the periodic arrays are coupled to the surface plasmon resonances of the rings (Purcell effect). Note, however, that the exact details of the enhancement depend on a number of experimental parameters that must be carefully analyzed. First, the intensity of the PL signal depends on the excitation rate of the emitters which is itself influenced by the presence of the metallic structures. This factor does not significantly vary from one periodic array to another since all the curves of Fig. 4(a) are bound by the same lower value of $\approx 2$ far from the plasmon resonance. Second, the spectral shape depends on the nature of the interactions between the QDs and the plasmonic antennas. Here the dielectric layer containing the PbS QDs is separated by a distance of $30 \mathrm{~nm}$ from the antennas so the emitters interact with the rings through their electromagnetic field-and not via nonradiative energy transfers such as those evidenced in [19]. Last, the measured boost in photoluminescence is also affected by the finite collection angle of our setup even though we have verified 
that the enhancement spectra are largely independent from this parameter for a series of objectives with N.A. varying from 0.1 to 0.9 (results not shown here).

Figure 4(a) also shows that the amplitude of the PL enhancement peak does not follow a monotonic behavior as the period varies, a result that can be explained by two competing factors. On one hand, the enhancement depends on the filling factor of the array: because the QDs are uniformly distributed above the structures, the number of emitters effectively coupled to the rings decreases with the period, resulting in a smaller PL enhancement. On the other hand, reducing the period broadens the plasmonic resonances, lowering their quality factor. Thus, there exists a tradeoff between these two opposite effects that results in an optimized enhancement of approximately 7 for a period of $600 \mathrm{~nm}$. This value is relatively modest because we work with thick luminescent layers $(\approx 100 \mathrm{~nm})$ in which the dots located far from the antennas interact less with them.

We next repeat the measurements at $4.2 \mathrm{~K}$ so as to increase the quantum yield of the $\mathrm{PbS} \mathrm{QDs}$ by an order of magnitude as previously discussed with Fig. 3. The PL enhancement spectra, plotted in Fig. 4(c), have essentially the same features as those recorded at room temperature except that their amplitude is smaller. This observation is consistent with the well-known fact that plasmonic antennas are less efficient when used in conjunction with bright emitters. In this low-loss limit, the part of electromagnetic radiation that is converted into heat in the metal significantly increases the number of absorption channels of the system, dampening the boost in photoluminescence produced by the antennas [1]. However, it is interesting to note that the absolute value of the PL enhancement has only been divided by approximately two even though the intrinsic quantum yield of the nanocrystals has been multiplied by ten. In addition, this enhancement is not only weaker at the plasmonic resonance wavelengths, where the losses by absorption are maximized, but also everywhere else. In fact, the curves of Figs. 4(a) and 4(c) are almost proportional, including at wavelengths far from the plasmon resonances, as is apparent in Fig. 4(d) where three representative spectra obtained at $4 \mathrm{~K}$ have been multiplied by a constant factor of 1.8 and superimposed with those obtained at $300 \mathrm{~K}$. These observations are reproducible if one cycles the temperature back and forth: The PL enhancement spectra fully recover their initial intensities when the sample is reheated and their shape does not change at intermediate temperatures.

The proportionality between the curves measured at room and cryogenic temperatures is the central result of this study. The apparent simplicity of this observation is deceiving for one reason-it is at odds with the intuitive picture that the magnitude of PL enhancement should be relatively independent of the intrinsic quantum yield at off-resonance wavelengths where absorption losses are relatively small. By the same token, it also contradicts the predictions of the two-level model that is often used to interpret such experiments on plasmonic antennas [34]. This behavior can be understood by the fact that the ring arrays preferentially favor, among the continuum of radiative transitions of the system, those that are matched with their plasmonic resonances. To test this hypothesis, we propose a qualitative model that accounts for the basic properties of our CQDs, namely, the existence of two emission lines as evidenced in Fig. 3 and an emission spectrum dominated by inhomogeneous broadening. In this model, each CQD is a source that emits light at two distinct frequencies $\omega_{f}$ and $\omega_{f}^{\prime}$ [34]. We note $\gamma_{r}$ and $\gamma_{r}^{\prime}\left(\Gamma_{r}\right.$ and $\left.\Gamma_{r}^{\prime}\right)$ the radiative decay rates of these transitions in the absence (in the presence) of the antennas and suppose that there is no correlation between the two channels. Under these assumptions, the power radiated by the $N$ sources of the system at a frequency $\omega_{f 0}$ can be formally written as a contribution from the two types of transitions:

$$
P\left(\omega_{f 0}\right)=k_{\mathrm{det}} \Gamma_{r}\left(\omega_{f 0}\right) I+k_{\mathrm{det}} \Gamma_{r}^{\prime}\left(\omega_{f 0}\right) I^{\prime},
$$

where $k_{\mathrm{det}}$ represents the response of the detector and $I$ and $I^{\prime}$ are integrals corresponding to the fraction of CQDs emitting through each type of transition at $\omega_{f 0}$. In the spectral range where photoluminescence occurs, the effect of the antennas on the emitters is a change in the decay rate due to the Purcell effect. Because the latter is solely determined by the local density of photonic states of the system, the decay rates of both channels are modified by the same factor $g\left(\omega_{f 0}\right)$ so Eq. (1) can be written as

$$
P\left(\omega_{f 0}\right)=k_{\operatorname{det}} g\left(\omega_{f 0}\right)\left[\gamma_{r}\left(\omega_{f 0}\right) I+\gamma_{r}^{\prime}\left(\omega_{f 0}\right) I^{\prime}\right] .
$$

Integrals $I$ and $I^{\prime}$ result from the inhomogeneous broadening of the $N$ emitters and are almost independent from the emission frequency [34]. Thus, Eq. (2) predicts that the PL signal is proportional to the radiative enhancement $g\left(\omega_{f 0}\right)$, a result that remains true if we normalize this expression by the signal $P_{0}$ produced in the absence of the antennas. It is also worth noting that this model ceases to provide a satisfying description of our experiments if one neglects the inhomogeneous broadening or if one suppresses one of the two radiative channels - in other words, our measurements cannot be understood if one treats our CQDs as two-level emitters. On the other hand, this simple model neglects potentially important effects such as the possibility that the plasmonic antennas may trigger a series of radiative transitions that are not allowed in a homogeneous environment.

Finally, a more critical examination of Fig. 4(d) reveals that the measurements at room and cryogenic temperatures are not exactly proportional. To explain this result, it is important to note that we have neglected the influence of the temperature on the $\mathrm{Au}$ rings themselves in the discussion. As documented in a few studies, the plasmonic properties of nanoparticles with significant surface roughness such as our lithographied rings are only marginally affected by the temperature, except for a slight narrowing and blue shifting of the peak as $T$ decreases [38]. This is such a spectral evolution that we observe in Fig. 4(d).

In conclusion, temperature-dependent experiments provide valuable information on the interactions between semiconducting nanocrystals and noble metal nanoparticle arrays. Our measurements indicate that the spectral signature of an inhomogeneous ensemble of nanocrystals coupled to plasmonic antennas is easy to predict-it is roughly the product of the PL spectrum of the emitters alone and the spectral signature of the localized plasmons, producing a boost in photoluminescence even in the case of bright CQDs with high quantum yields. Although this result may appear rather intuitive at first, we 
have shown that it cannot be explained with simple arguments. It is the consequence of the inhomogeneous broadening of the emitters considered in this study and the fact that they emit light through at least two distinct channels.

We would like to thank Maria Tchernycheva and Pierre Lavenus for their assistance with the cryogenic experiments.
Financial support was provided by the RTRA Triangle de la Physique under Grant No. 2010-008T, by Université Paris-Sud under Grant Attractivité 2010 and by the Labex NanoSaclay. This work was partly supported by the french RENATECH network. The sample fabrication has been performed at the nanocenter CTU-IEF-Minerve, which was partially funded by the Conseil Général de l'Essonne.
[1] P. Bharadwaj, B. Deutsch, and L. Novotny, Adv. Opt. Photon. 1, 438 (2009).

[2] M. Thomas, J.-J. Greffet, R. Carminati, and J. R. AriasGonzalez, Appl. Phys. Lett. 85, 3863 (2004).

[3] S. Kühn, U. Håkanson, L. Rogobete, and V. Sandoghdar, Phys. Rev. Lett. 97, 017402 (2006).

[4] P. Anger, P. Bharadwaj, and L. Novotny, Phy. Rev. Lett. 96, 113002 (2006).

[5] A. Mohammadi, V. Sandoghdar, and M. Agio, New J. Phys. 10, 105015 (2008).

[6] O. L. Muskens, V. Giannini, J. A. Sánchez-Gil, and J. Gómez Rivas, Nano Lett. 7, 2871 (2007).

[7] L. Rogobete, F. Kaminski, M. Agio, and V. Sandoghdar, Opt. Lett. 32, 1623 (2007).

[8] A. Kinkhabwala, Z. Yu, S. Fan, Y. Avlasevich, K. Mullen, and W. E. Moerner, Nat Photon. 3, 654 (2009).

[9] H. Aouani, S. Itzhakov, D. Gachet, E. Devaux, T. W. Ebbesen, H. Rigneault, D. Oron, and J. Wenger, ACS Nano 4, 4571 (2010).

[10] R. Esteban, T. V. Teperik, and J. J. Greffet, Phys. Rev. Lett. 104, 026802 (2010).

[11] H. Gersen, M. F. García-Parajó, L. Novotny, J. A. Veerman, L. Kuipers, and N. F. van Hulst, Phys. Rev. Lett. 85, 5312 (2000).

[12] T. H. Taminiau, F. D. Stefani, F. B. Segerink, and N. F. van Hulst, Nat Photon. 2, 234 (2008).

[13] A. G. Curto, G. Volpe, T. H. Taminiau, M. P. Kreuzer, R. Quidant, and N. F. van Hulst, Science 329, 930 (2010).

[14] G. Ritchie, E. Burstein, and R. B. Stephens, J. Opt. Soc. Am. B 2, 544 (1985).

[15] J. Kümmerlen, A. Leitner, H. Brunner, F. R. Aussenegg, and A. Wokaun, Mol. Phys. 80, 1031 (1993).

[16] T. D. Corrigan, S. Guo, R. J. Phaneuf, and H. Szmacinski, J. Fluoresc. 15, 777 (2005).

[17] R. M. Bakker, V. P. Drachev, Z. Liu, H.-K. Yuan, R. H. Pedersen, A. Boltasseva, J. Chen, J. Irudayaraj, A. V. Kildishev, and V. M. Shalaev, New J. Phys. 10, 125022 (2008).

[18] D. M. Koller, U. Hohenester, A. Hohenau, H. Ditlbacher, F. Reil, N. Galler, F. R. Aussenegg, A. Leitner, A. Trügler, and J. R. Krenn, Phys. Rev. Lett. 104, 143901 (2010).

[19] P. Viste, J. Plain, R. Jaffiol, A. Vial, P. M. Adam, and P. Royer, ACS Nano 4, 759 (2010).

[20] S. R. K. Rodriguez, G. Lozano, M. A. Verschuuren, R. Gomes, K. Lambert, B. De Geyter, A. Hassinen, D. Van Thourhout, Z. Hens, and J. Gómez Rivas, Appl. Phys. Lett. 100, 111103 (2012).
[21] M. Ringler, A. Schwemer, M. Wunderlich, A. Nichtl, K. Kürzinger, T. A. Klar, and J. Feldmann, Phys. Rev. Lett. 100, 203002 (2008).

[22] M. Munsch, A. Mosset, A. Auffèves, S. Seidelin, J. P. Poizat, J.-M. Gérard, A. Lemaitre, I. Sagnes, and P. Senellart, Phys. Rev. B 80, 115312 (2009).

[23] V. I. Klimov, Annu. Rev. Phys. Chem. 58, 635 (2007).

[24] H. M. Sevian and J. L. Skinner, Theor. Chim. Acta 82, 29 (1992).

[25] R. F. Kubin and A. N. Fletcher, J. Luminesc. 27, 455 (1982).

[26] K. Maruszewski, D. Andrzejewski, and W. Strek, J. Luminesc. 72-74, 226 (1997)

[27] G. W. Walker, V. C. Sundar, C. M. Rudzinski, A. W. Wun, M. G. Bawendi, and N. G. Nocera, Appl. Phys. Lett. 83, 3555 (2003).

[28] J. Aizpurua, P. Hanarp, D. S. Sutherland, M. Käll, G. W. Bryant, and F. J. García de Abajo, Phys. Rev. Lett. 90, 057401 (2003).

[29] A. Mary, D. M. Koller, A. Hohenau, J. R. Krenn, A. Bouhelier, and A. Dereux, Phys. Rev. B 76, 245422 (2007).

[30] L. Zhao, K. L. Kelly, and G. C. Schatz, J. Phys. Chem. B 107, 7343 (2003).

[31] L. Turyanska, A. Patane, M. Henini, B. Hennequin, and N. R. Thomas, Appl. Phys. Lett. 90, 101913 (2007).

[32] F. Yue, J. W. Tomm, and D. Kruschke, Phys. Rev. B 87, 195314 (2013).

[33] S. A. Crooker, T. Barrick, J. A. Hollingsworth, and V. I. Klimov, Appl. Phys. Lett. 82, 2793 (2003).

[34] See Supplemental Material at http://link.aps.org/supplemental/ 10.1103/PhysRevB.91.085412 for more details on the fits performed on Fig. 3 and for a more technical discussion on how to obtain Eqs. (1) and (2).

[35] M. S. Gaponenko, A. A. Lutich, N. A. Tolstik, A. A. Onushchenko, A. M. Malyarevich, E. P. Petrov, and K. V. Yumashev, Phys. Rev. B 82, 125320 (2010).

[36] B. Ullrich, J. S. Wang, and G. J. Brown, Appl. Phys. Lett. 99, 081901 (2011).

[37] S. Pichler, T. Rauch, R. Seyrkammer, M. Boberl, S. F. Tedde, J. Furst, M. V. Kovalenko, U. Lemmer, O. Hayden, and W. Heiss, Appl. Phys. Lett. 98, 053304 (2011).

[38] J.-S. G. Bouillard, W. Dickson, D. P. O’Connor, G. A. Wurtz, and A. V. Zayats, Nano Lett. 12, 1561 (2012). 